\title{
ELEMENTARY AMENABLE SUBGROUPS OF R. THOMPSON'S GROUP $F$
}

\author{
MATTHEW G. BRIN
}

\section{INTRODUCTION}

This paper contributes to the study of the subgroups of Thompson's group $F$ by constructing a sequence of subgroups of increasing complexity. The group $F$ is an interesting finitely presented group with a pleasant, faithful representation in the group $P L_{o}(I)$ of orientation preserving, piecewise linear, self homeomorphisms of the unit interval. Thus our subgroups also lie in $P L_{o}(I)$. The subgroups we construct are all elementary amenable of a certain bounded degree (see Section 1.2 for definitions) while $F$ is not elementary amenable. Thus the complexities of the subgroups that we construct are in some sense strictly less than that of $F$ itself and we raise the question of whether there are subgroups of $P L_{o}(I)$ that are in the gap between the groups we construct and the group $F$. We also raise (and give specific meaning to) the question of whether $F$ is the "only" subgroup of $P L_{o}(I)$ that is not elementary amenable. See Conjecture 3

The group $F$ originated with Thompson in his studies in algebraic logic [29], was independently discovered as the key behind interesting examples in shape and homotopy theory [14, 13, 15], can be called the "structure group" of the associative law [12], and arises in a fundamental way in the theory of diagram groups [20. It is also the source of difficult questions, the most prominent of which is whether $F$ is amenable [16. See 8] and [5] Section 4] for an introduction to Thompson's groups.

1.1. Background. The subgroup structure of $F$ is only partly understood. Some information about subgroups of $F$ comes from the faithful representation of $F$ in $P L_{o}(I)$. For example, $P L_{o}(I)$ is torsion free and no subgroup of $P L_{o}(I)$ can be free on two generators (4] or [8]). Further, every non-abelian subgroup of $P L_{o}(I)$ contains a copy of $\mathbf{Z}\{\mathbf{Z}$, the wreath product of $\mathbf{Z}$ with itself [19] Theorem 21]. Other information comes from the fact that $F$ is (one of the prime examples of) a diagram group [20. For example, every nilpotent subgroup of $F$ is abelian [19, Cor. 15] . (One can also show that every nilpotent subgroup of $P L_{o}(I)$ is abelian.)

Other results that will be reflected in the themes of this paper are that $F$ contains many copies of itself as well as many wreath products [2, and in particular contains the iterated wreath product $(\cdots((\mathbf{Z} \backslash \mathbf{Z}) \prec \mathbf{Z}) \prec \cdots)$ [19, Cor. 20]. Not relevant to this paper are various results about the metric structure of subgroups of $F$. See [19. Section 6], 7], 6] and [10].

Date: June 29, 2004.

AMS Classification (2000): primary 20E07, secondary 20F19, 20F22, 20E22. 
1.2. The classes $E G_{\alpha}, E G, A G$ and $N F$. Thompson's group and its subgroups are scattered among several classes of groups from [11]: the class $E G$ of elementary amenable groups, the class $A G$ of amenable groups, and the class $N F$ of groups with no subgroup isomorphic to the free group on two generators. The class $N F$ needs no further definitions, the definitions relevant to the class $E G$ will be given below, and the definitions relevant to the class $A G$ are to be found in 11, 30. or 31 as well as full discussions on the relations between the classes. It is known (11], 31]) that $E G \subseteq A G \subseteq N F$, that both containments are proper (26], 1] and [17]), and that there are finitely presented groups in each difference ([18] and [27]).

Let $E G_{0}$ be the class of all groups that are either finite or abelian. The class $E G$ is defined in [11] as the smallest class of groups that contains $E G_{0}$ and is closed under the four operations of (I) taking subgroups, (II) taking quotients, (III) taking extensions and (IV) taking direct limits. It is shown in 9] operations (I) and (II) are not necessary. We need some details of this.

A hierarchy $E G_{\alpha}$ of classes of groups indexed by the ordinals is defined inductively in 9 as follows. With $E G_{0}$ as defined above, let $\alpha>0$ be an ordinal so that $E G_{\beta}$ is defined for all $\beta<\alpha$. If $\alpha$ is not a limit ordinal, then $E G_{\alpha}$ is the class of all groups $G$ that are extensions

$$
1 \rightarrow N \rightarrow G \rightarrow Q \rightarrow 1
$$

with $N$ and $Q$ in some $E G_{\beta}$ with $\beta<\alpha$, or are the direct limits of groups from the various $E G_{\beta}$ with $\beta<\alpha$. If $\alpha$ is a limit ordinal, then $E G_{\alpha}$ is the union of all the $E G_{\beta}$ with $\beta<\alpha$. The following combines Propositions 2.1 and 2.2 of 9 .

Proposition 1.1 (Chou). The following are true of the items defined above.

(a) Each $E G_{\alpha}$ is closed under operations (I) and (II).

(b) $E G$ is the union of the $E G_{\alpha}$.

(c) $E G$ is the smallest class of groups that contains $E G_{0}$ and is closed under operations (III) and (IV).

It is remarked in [9] that since the class of isomorphism types of finitely generated groups has the cardinality of the continuum, there must be some $E G_{\delta}$ containing all finitely generated groups in $E G$, and since every group is the direct limit of its finitely generated subgroups, we must have $E G=E G_{\delta+1}$.

If $G$ is a group in $E G$, then the elementary class of $G$ (often referred to in this paper as the class of $G$ ) is the smallest ordinal $\alpha$ so that $G$ is in $E G_{\alpha}$.

We use standard notation for certain ordinals. The ordinal $\omega$ is the smallest infinite ordinal, setting $1 \omega=\omega$ gives the definition of $(n+1) \omega$ for $n \geq 1$ as the limit of $n \omega, n \omega+1, n \omega+2, \ldots$, and $\omega^{2}$ as the limit of $1 \omega, 2 \omega, 3 \omega, \ldots$.

1.3. Results and questions. The main result of this paper is the following.

Theorem 1. For each non-limit ordinal $\alpha \leq \omega^{2}+1$, there is a subgroup of $F$ that is of elementary class $\alpha$.

We obtain Theorem 1 by finding techniques for building groups of higher class from groups of a given class or set of classes. We observe that these techniques work well as long as we are working primarily with finitely generated groups and that our technique runs out of finitely generated groups at class $\omega^{2}+1$. The question arises as to whether it is possible to get past $\omega^{2}+1$ in either $F$ or $P L_{o}(I)$. 
It is remarked above that $P L_{o}(I)$ is in the class $N F$ and it is known [8] that $F$ is not in $E G$. It is very easy [2] for isomorphic copies of $F$ to appear in subgroups of $P L_{o}(I)$. While not stated as such, our constructions are designed to increase elementary class while avoiding the inclusion of copies of $F$.

We now formally bundle the various observations that we have made above, the theorems of this paper, and the results of [2] and [19 into various questions and conjectures.

The observation of [19] that every non-abelian subgroup of $P L_{o}(I)$ contains a copy of $\mathbf{Z} \imath \mathbf{Z}$ has the form of an "alternative" theorem: every subgroup of $P L_{o}(I)$ is either abelian or contains $\mathbf{Z} \imath \mathbf{Z}$ as a subgroup. The group $(\cdots((\mathbf{Z} \imath \mathbf{Z}) \imath \mathbf{Z}) \imath \cdots)$, which we will denote by $(\mathbf{Z} \text { ? })^{\infty}$, is not solvable, while all finite iterations of the wreath product of copies of $\mathbf{Z}$ are solvable. Sapir has raised the question of whether every non-solvable subgroup of $F$ contains a copy of $(\mathbf{Z} \imath)^{\infty}$. The question can also be asked of $P L_{o}(I)$. Our second result concerns another non-solvable wreath product

$$
\infty(\imath \mathbf{Z})=(\cdots \curlywedge(\mathbf{Z} \succ(\mathbf{Z} \imath \mathbf{Z})) \cdots)
$$

where the wreath product is that of permutation groups (see Section 2 for definitions) and not the standard (restricted) wreath product of [25].

Theorem 2. Both $\infty(\imath \mathbf{Z})$ and $(\mathbf{Z} \imath)^{\infty}$ are isomorphic to subgroups of $F$. Further, there is no subgroup of $(\mathbf{Z} \imath)^{\infty}$ that is isomorphic to ${ }^{\infty}(\zeta \mathbf{Z})$, and there is no subgroup of $\infty(\imath \mathbf{Z})$ that is isomorphic to $(\mathbf{Z} \imath)^{\infty}$.

From Theorem 2] the answer to Sapir's question is "No." However, the following can be asked (which we put in "alternative" form).

Question 1. Is it true of every subgroup of $P L_{o}(I)$ that it is either solvable or it contains a subgroup that is isomorphic to one of ${ }^{\infty}(\imath \mathbf{Z})$ or $(\mathbf{Z} \imath)^{\infty}$ ?

The next question relates to our failure to find elementary amenable subgroups of class greater than $\omega^{2}+1$.

Question 2. Is $\omega^{2}+1$ the largest elementary class of an elementary amenable subgroup of $P L_{o}(I)$ ?

The following has been privately suggested by the author.

Conjecture 3. Every subgroup of $P L_{o}(I)$ is either elementary amenable or it contains a subgroup that is isomorphic to $F$.

If we raise Question 2 to the status of conjecture and combine it with Conjecture 3 we get the following.

Conjecture 4. Every subgroup of $P L_{o}(I)$ is either elementary amenable of some class $\alpha$ with $\alpha \leq \omega^{2}+1$, or it contains a subgroup that is isomorphic to $F$.

We include a remark that has little to do with this paper.

The group $F$ is contained in $N F \backslash E G$, but it is not known whether it is contained in $A G$. The group $F$ has a presentation that is somewhat smaller than that of the example in [18] of a group in $A G \backslash E G$ and that is vastly smaller than that of the example in 27] of a group in $N F \backslash A G$. Thus it is still of interest to locate $F$ accurately in the string of classes $E G \subseteq A G \subseteq N F$. It has also been put forth by Grigorchuk that answering the question of the amenability of $F$ will be an important step in understanding the groups in the class $N F$. 
1.4. The Thompson group $V$. Thompson's group $V$ (see [ [8]) is more flexible in that it acts on the totally discontinuous Cantor set. In particular it contains copies of the free group on two generators. It will be clear to those familiar with $V$, that there is much more room to apply the constructions of this paper in the setting of $V$. It would be of interest to know more about the subgroups of $V$. That this is not a trivial task is indicated by the fact that $V$ contains a copy of the infinite symmetric group and thus a copy of every finite group. It would also be of interest to know if there is a criterion along the lines of [2] to recognize $V$ among the homeomorphisms of the Cantor set.

1.5. Section contents. In Section 2 we develop construction techniques that will apply to the setting of $P L_{o}(I)$. Our main tool will be the permutation wreath product and we set up methods for recognizing the product. Section 3 discusses methods for recognizing the elementary class of groups with emphasis on recognizing the elementary class of wreath products. Section 4 proves Theorem 1 while Section 5 proves Theorem 2

In the narrative below, lemmas without proofs are to be taken as exercises.

1.6. Thanks. The author would like to thank Fernando Guzmán for numerous helpful conversations.

\section{Construction techniques}

2.1. Permutation groups. A permutation group is a pair $(G, Z)$ in which $Z$ is a set and $G$ is a subgroup of $\operatorname{Sym}(Z)$, the symmetric group on $Z$ or the group of all bijections from $Z$ to itself. We have no need of a more general notion.

The group $G$ will act on $Z$ on the right and $x G=\{x g \mid g \in G\}$ is the orbit of $x$ under $G$. The support of $G$ written $\operatorname{Supp}(G)$ is the set $\{x \mid\{x\} \neq x G\}$. There is also an action of $G$ on the subsets of $Z$ in the usual way: if $X$ is a subset of $Z$, then $X g$ denotes the image of $X$ under the action of $g \in G$ and is the set $\{x g \mid x \in X\}$. Note that this makes $X G$ a collection of sets.

2.2. The permutation wreath product. We need to recognize the wreath product of two permutation groups. If $(G, A)$ and $(H, B)$ are permutation groups in which the action of $H$ on $B$ is transitive, then the following definition of the permutation group $(G \imath H, A \times B)$ is equivalent to the definition found in 28] Section 1.6]. We define $G ? H$ as the subgroup of $\operatorname{Sym}(A \times B)$ generated by $G^{\prime}$ and $H^{*}$ where $G^{\prime}$ and $H^{*}$ are images of two homomorphisms $G \rightarrow G^{\prime}$ and $H \rightarrow H^{*}$. The first homomorphism is defined by choosing a fixed $b_{1} \in B$ and sending $g \in G$ to $g^{\prime} \in G^{\prime}$ where for and $(a, b) \in A \times B$,

$$
(a, b) g^{\prime}= \begin{cases}(a g, b), & b=b_{1}, \\ (a, b), & b \neq b_{1},\end{cases}
$$

and the second is defined by sending $h \in H$ to $h^{*} \in H^{*}$, where $(a, b) h^{*}=(a, b h)$. The definition does not depend on the choice of $b_{1}$.

In the event that the action of $H$ on $B$ is not transitive, then the permutation wreath product as defined in 28] is not generated by $H^{*}$ and $G^{\prime}$, but instead by $H^{*}$ and an isomorphic copy of $G$ for each orbit in $B$ under the action of $H$. In our setting, we do not have to worry about this. Note that we do not require that the action of $G$ on $A$ be transitive. 
The reader can glean the properties of the permutation wreath product from 28] including the associativity of the product which is reflected in Corollary 2.5.1 below.

2.3. Pre-wreath structures. The wreath product involves two factors. To make it easier to iterate the product, we give conditions under which it is easy to be a factor.

Definition 2.1. A pre-wreath structure is a quadruple $(Z, Y, H, X)$ in which $H$ is a non-trivial group and the rest are sets and the following are satisfied.

(1) $H \subseteq \operatorname{Sym}(Z)$

(2) $\operatorname{Supp}(H) \subseteq Y \subseteq Z$.

(3) $\emptyset \neq X \subseteq Y$.

(4) For all $\bar{h} \in H$, we have that $X h \cap X \neq \emptyset$ implies $\left.h\right|_{X}=\left.1\right|_{X}$.

(5) For all $1 \neq h \in H$, there is a $j \in H$ so that $X j h \neq X j$.

We have no need of trivial groups here and the non-triviality assumption on $H$ will be convenient. The need for having both $Y$ and $Z$ will not be apparent until Proposition 2.5 below.

The collection $\{X h \mid h \in H\}$ will be important and we will denote it by $X H$. We will use $\overline{X H}$ to denote the union $\bigcup_{h \in H} X h$ of the elements in $X H$.

Lemma 2.2. Let $(Z, Y, H, X)$ be a pre-wreath structure. Then the following hold.

(a) $X H$ is a collection of pairwise disjoint non-empty subsets of $Y$.

(b) $X \in X H$.

(c) For each $h \in H$ and $A \in X H$ we have $A h \in X H$.

(d) Each non-identity element $h \in H$ has some $A \in X H$ so that $A h \neq A$.

(e) For every $A$ and $B$ in $X H$, there is an $h \in H$ with $A h=B$.

(f) For each $h, k \in H$ and $A \in X H$, if $A h=A k$, then $\left.h\right|_{A}=\left.k\right|_{A}$.

From (c), there is a homomorphism $H \rightarrow \operatorname{Sym}(X H)$. Item (d) implies that this homomorphism is an injection. In particular $(H, X H)$ is a permutation group if we identify $H$ with its image under the injection $H \rightarrow \operatorname{Sym}(X H)$.

Other observations are that (e) implies that the action of $H$ on $X H$ is transitive, and (f) implies that for any $A$ and $B$ in $X H$, there is only one function from $A$ to $B$ that is a restriction of an element of $H$.

In a pre-wreath structure $(Z, Y, H, X)$, we call $X H$ the carrier of the structure.

Lemma 2.3. If $(Z, Y, H, X)$ is a pre-wreath structure, and if $X^{\prime}$ is a non-empty subset of $X$, then $\left(Z, Y, H, X^{\prime}\right)$ is a pre-wreath structure.

2.4. Pre-wreath structures to wreath products. We need a standard fact.

Lemma 2.4. If $G$ and $H$ are subgroups of a group $M$, then any element of $\langle G, H\rangle$ can be written as an element of $H$ followed by a product of elements of $G$ each of which is conjugated by an element of $H$. 
Proof. We have

$$
\begin{aligned}
& h_{n} g_{n-1} \cdots h_{3} g_{2} h_{2} g_{1} h_{1}= \\
& \left(h_{n} \cdots h_{3} h_{2} h_{1}\right) \\
& \left(h_{1}^{-1} h_{2}^{-1} h_{3}^{-1} \cdots h_{n-1}^{-1} g_{n-1} h_{n-1} \cdots h_{3} h_{2} h_{1}\right) \\
& \cdots \\
& \quad\left(h_{1}^{-1} h_{2}^{-1} h_{3}^{-1} g_{3} h_{3} h_{2} h_{1}\right) \\
& \quad\left(h_{1}^{-1} h_{2}^{-1} g_{2} h_{2} h_{1}\right) \\
& \left(h_{1}^{-1} g_{1} h_{1}\right) .
\end{aligned}
$$

Proposition 2.5. Let $(Z, Y, H, X)$ and $(Z, X, G, W)$ be pre-wreath structures. Then

$$
(Z, Y,\langle G, H\rangle, W)
$$

is a pre-wreath structure and its carrier $W\langle G, H\rangle$ is equal to

$$
W G H=\{W g h \mid g \in G, h \in H\} .
$$

Further, the permutation group $(\langle G, H\rangle, W G H)$ is isomorphic to (similar to) the permutation group $(G \imath H,(W G) \times(X H))$ as defined in 28].

The picture below might help understand the hypotheses of Proposition 2.5. We only indicate $Y$ and some of its subsets since $Y$ contains all of the "action."

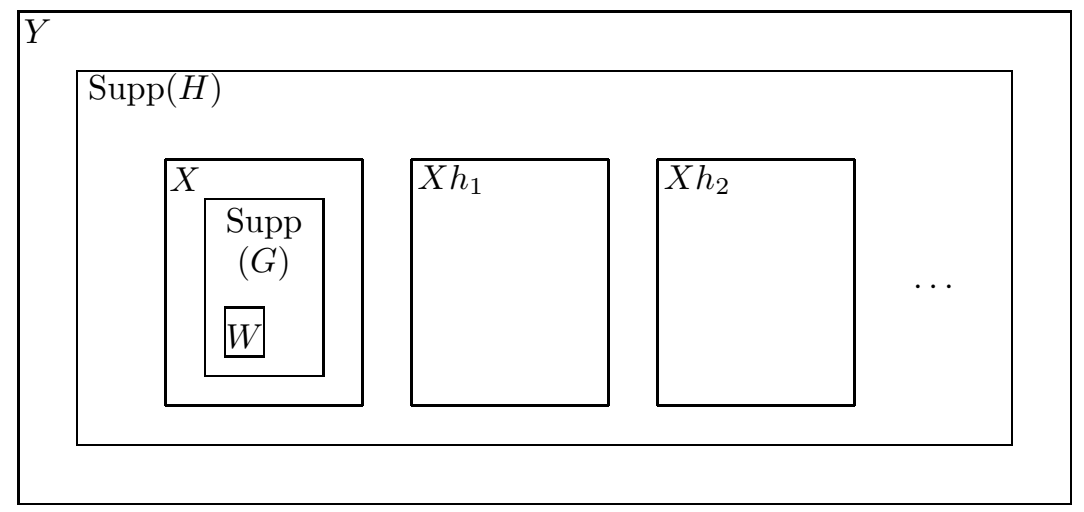

In the following proof, (1)-(5) refer to items in Definition 2.1 and (a)-(f) refer to items in Lemma 2.2

Proof. Denote $\langle G, H\rangle$ by $M$. The group $M$ is contained in $\operatorname{Sym}(Z)$ with support in $Y$. We have $\emptyset \neq W \subseteq X \subseteq Y$. Thus (1), (2) and (3) are obtained with no problem.

For the rest of the proof, we need a better understanding of the group $M$. Let $w \in M$. By Lemma 2.4 $w$ is an element of $H$ followed by a product of elements of $G$ conjugated by elements of $H$.

Note that the conjugate $G^{h}=h^{-1} G h$ has support in $X h$. Because of (4) applied to $(Z, Y, H, X)$, we know that the conjugate $G^{h}$ is completely determined by the set $X h$. Since the elements $X h$ of $X H$ are pairwise disjoint, the group

$$
K=\left\langle G^{h} \mid h \in H\right\rangle
$$


is the direct sum of the elements of $\left\{G^{h} \mid h \in H\right\}$. By (f) applied to $(Z, Y, H, X)$, the action of $H$ on $K$ is to permute the direct factors and we get that $M$ is the semidirect product $K \rtimes H$.

Consider $W w$ with $w \in M$. We know $w=h v$ with $h \in H$ and $v \in K$, so $W w=W h v \subseteq X h v$. All components of $v$ in the product structure of $K$ fix $X h$ except the component coming from $G^{h}$ which has support in $X h$. Thus $W w \subseteq X h$.

If $W w \cap W w^{\prime} \neq \emptyset$ with $w=h v$ and $w^{\prime}=h^{\prime} v^{\prime}$, then $X h \cap X h^{\prime} \neq \emptyset$ and $\left.h\right|_{W}=\left.h^{\prime}\right|_{W}$ since $\left.h\right|_{X}=\left.h^{\prime}\right|_{X}$ and $W \subseteq X$. Now the actions of $v$ and $v^{\prime}$ on $X h=X h^{\prime}$ are determined by their components from $G^{h}=G^{h^{\prime}}$. Let $h^{-1} g h$ and $h^{-1} g^{\prime} h$ be these components from $v$ and $v^{\prime}$, respectively. It follows that $W g \cap W g^{\prime} \neq \emptyset$, so $\left.g\right|_{W}=\left.g^{\prime}\right|_{W}$. Since $W \subseteq X$ and $\left.h\right|_{X}=\left.h^{\prime}\right|_{X}$, we get that $\left.(h g)\right|_{W}=\left.\left(h^{\prime} g^{\prime}\right)\right|_{W}$.

Let $1 \neq w=h v$ be from $M$. If $h \neq 1$, then there is a $j \in M$ so that $X j h \neq X j$. Now $W j \subseteq X j$ has $W j w \subseteq X j h v=X j h$ which is disjoint from $X j$. If $h=1$, then $v \neq 1$ and some component $l^{-1} g l$ of $v$ is not trivial. There is a $k$ from $G$ with $W k g \neq W k$. Now $W k l w=W k l v=W k l\left(l^{-1} g l\right)=W k g l \neq W k l$ since $l$ is a bijection. This verifies that $(Z, Y, M, W)$ is a pre-wreath structure.

That $W G H \subseteq W\langle G, H\rangle=W M$ is clear. If $w \in M$ is of the form $w=h v$ with $h \in H$ and $v \in K$, then let $h^{-1} g h$ be the component of $v$ from $G^{h}$. Now $W w=W h v=W h\left(h^{-1} g h\right)=W g h$ and we have $W M \subseteq W G H$.

To establish the claimed similarity in the last statement, we note that sending $(W g, X h) \in(W G) \times(X H)$ to $W g h \subseteq X h$ is clearly a surjection from $(W G) \times(X H)$ onto $W G H$. It is one-to-one since there is only one bijection from $X$ to $X h$ that is a restriction of an element of $H$. Use $t$ to denote the bijection $(W g, X h) \mapsto W g h$.

We note that $M$ is defined as $M=\langle G, H\rangle$ in $\operatorname{Sym}(Z)$. Since (d) holds for the pre-wreath structure $(Z, Y, M, W)$, we know that the image of $M$ in $\operatorname{Sym}(W G H)$ is isomorphic to $M$. Since $M$ is generated by $G$ and $H$, its image in $\operatorname{Sym}(W G H)$ is generated by the images of $G$ and $H$. We will now use $M, G$ and $H$ to denote their images in $\operatorname{Sym}(W G H)$.

The group $G$ ? $H$ is the subgroup of $\operatorname{Sym}((W G) \times(X H))$ generated by $G^{\prime}$ and $H^{*}$ where $g \in G$ is sent to $g^{\prime} \in G^{\prime}$ and $h \in H$ is sent to $h^{*} \in H^{*}$, and where for and $\left(W g_{1}, X h_{1}\right) \in(W G) \times(X H)$,

$$
\left(W g_{1}, X h_{1}\right) g^{\prime}= \begin{cases}\left(W g_{1} g, X h_{1}\right), & X h_{1}=X \\ \left(W g_{1}, X h_{1}\right), & X h_{1} \neq X\end{cases}
$$

and $\left(W g_{1}, X h_{1}\right) h^{*}=\left(W g_{1}, X h_{1} h\right)$.

Now

$$
\begin{aligned}
\left(W g_{1}, X h_{1}\right) t g t^{-1}=\left(W g_{1} h_{1}\right) g t^{-1} & = \begin{cases}\left(W g_{1} g\right) t^{-1}, & X h_{1}=X \\
\left(W g_{1} h_{1}\right) t^{-1}, & X h_{1} \neq X\end{cases} \\
& = \begin{cases}\left(W g_{1} g, X\right), & X h_{1}=X \\
\left(W g_{1}, X h_{1}\right), & X h_{1} \neq X\end{cases}
\end{aligned}
$$

Also

$$
\left(W g_{1}, X h_{1}\right) t h t^{-1}=\left(W g_{1} h_{1}\right) h t^{-1}=\left(W g_{1} h_{1} h\right) t^{-1}=\left(W g_{1}, X h_{1} h\right)
$$

and we see that $t$ conjugates the actions of $G$ and $H$ to those of $G^{\prime}$ and $H^{*}$. This establishes the similarity and completes the proof. 
Corollary 2.5.1. Let

$$
\begin{aligned}
& \left(Z, Y_{1}, H_{1}, Y_{0}\right), \\
& \left(Z, Y_{2}, H_{2}, Y_{1}\right), \\
& \left(Z, Y_{3}, H_{3}, Y_{2}\right)
\end{aligned}
$$

be pre-wreath structures and let $A=Y_{0} H_{1} \times Y_{1} H_{2} \times Y_{2} H_{3}$. Then

$$
\left(Z, Y_{3},\left\langle H_{1}, H_{2}, H_{3}\right\rangle, Y_{0}\right)
$$

is a pre-wreath structure with carrier

$$
T=Y_{0}\left\langle H_{1}, H_{2}\right\rangle H_{3}=Y_{0}\left\langle H_{1}, H_{2}, H_{3}\right\rangle=Y_{0} H_{1}\left\langle H_{2}, H_{3}\right\rangle=Y_{0} H_{1} H_{2} H_{3}
$$

and the permutation group $\left(\left\langle H_{1}, H_{2}, H_{3}\right\rangle, T\right)$ is isomorphic to (similar to)

$$
\left(\left(H_{1} \prec H_{2}\right) \prec H_{3}, A\right) \simeq\left(H_{1} \prec\left(H_{2} \prec H_{3}\right), A\right) .
$$

In Proposition 2.5 the status of $\langle G, H\rangle$ as a wreath product needs much less than was assumed. We leave it to the reader to extract a proof of the following from the proof of Proposition 2.5

Lemma 2.6. Let $(Z, Y, H, X)$ be a pre-wreath structure, and let $(G, Z)$ be a permutation group with support in $X$. Then restricting the action of $\langle G, H\rangle$ to $\overline{X H}$ gives a permutation group $(\langle G, H\rangle, \overline{X H})$ that is isomorphic to (similar to) the permutation group $(G \imath H, X \times(X H))$.

2.5. Homomorphisms and normal subgroups. There is much known about normal subgroups in wreath products [25]. We need very little and give below what we need. We start with a useful homomorphism.

Lemma 2.7. In the setting of Lemma 2.6, there is a homomorphism from $\langle G, H\rangle$ to $H$ that is the identity on $H$ and whose kernel is the group $K$ generated by the conjugates of $G$ in $\left\{G^{h} \mid h \in H\right\}$.

Proof. By Lemma 2.4] any element $w$ of $\langle G, H\rangle$ can be written as an element $h$ of $H$ multiplied by conjugates of elements of $G$. The conjugates of elements of $G$ have supports in the elements of $X H$, so $w$ and $h$ agree on $Z \backslash \overline{H X}$ and affect the same permutation on the sets of $X H$. But Lemma $2.2(\mathrm{f})$ says that an element of $H$ is determined by its permutation on $X H$ and its action on $Z \backslash \overline{H X}$. Thus sending $w$ to $h$ is well defined. It is clearly a homomorphism and its kernel is $K$.

The next lemma gives some control over the kernels and quotients.

Lemma 2.8. Assume the setting of Lemma 2.6 and let $N$ be a normal subgroup of $\langle G, H\rangle$.

(I) If $N$ contains an element whose permutation on $X H$ is non-trivial, then there is a subgroup of $N$ that surjects onto $G$.

(II) If every element of $N$ has trivial permutation on $X H$, then $\langle G, H\rangle / N$ surjects onto $H$.

Proof. Under the assumptions of (I), the transitivity of the action of $H$ on $X H$ gives an $f$ in $N$ so that $X f \neq X$, implying that $X f \cap X=\emptyset$. If $g$ is any element of $G$, then $f^{-1} g^{-1} f g$ is an element of $N$, its support is in $X \cup X f$, its restriction to $X$ equals that of $g$, and its restriction to $X f$ is that of $f^{-1} g^{-1} f$. This is a non-homomorphic injection of $G$ into $G \times G^{f}$ whose image we will call $\bar{G}$. The 
subgroup of $G \times G^{f}$ generated by $\bar{G}$ is a subgroup of $N$ whose support is in $X \cup X f$ and whose restriction to $X$ is $G$.

Under the assumptions of (II), it follows from Lemma 2.4 and the injection of $H$ into the symmetric group on $X H$, that every element of $N$ is a product of conjugates of $G$. Thus $N$ is contained in the kernel $K$ of Lemma 2.7 and the result follows from that lemma.

2.6. An infinite construction. Let $\left(Z, Y_{i}, H_{i}, Y_{i-1}\right)$ be pre-wreath structures for positive integers $i$. Let

$$
H_{i}^{\prime}=\left\langle H_{1}, H_{2}, H_{3}, \cdots, H_{i}\right\rangle
$$

We have $H_{i}^{\prime} \subseteq H_{i+1}^{\prime}$ and we can let $H$ be the union of all the $H_{i}^{\prime}$. We also have $Y_{i} \subseteq Y_{i+1}$ and we can let $Y$ be the union of all the $Y_{i}$. Let $T_{i}^{\prime}=Y_{0} H_{1} H_{2} H_{3} \cdots H_{i}$. Inductively from Proposition [2.5] and its corollary, each $\left(Z, Y_{i}, H_{i}^{\prime}, Y_{0}\right)$ is a prewreath structure, $T_{i}^{\prime}$ is its carrier and $T_{i}^{\prime}=Y_{0} H_{i}^{\prime}$. Clearly, $T_{i}^{\prime} \subseteq T_{i+1}^{\prime}$ and we let $T$ be the union of all the $T_{i}^{\prime}$.

Lemma 2.9. The quadruple $\left(Z, Y, H, Y_{0}\right)$ is a pre-wreath structure with carrier $T$.

Proof. We check (1)-(5) in Definition 2.1 to show that $\left(Z, Y, H, Y_{0}\right)$ is a pre-wreath structure. Items (1) and (3) are immediate. For (2), we note that an element of $H$ is in one of the $H_{i}^{\prime}$ and has its support in $Y_{i} \subseteq Y$. Items (4) and (5) are also immediate from the fact that each element of $H$ is in one of the $H_{i}^{\prime}$, and $\left(Z, Y_{i}, H_{i}^{\prime}, Y_{0}\right)$ is a pre-wreath structure by an inductive extension of Corollary 2.5.1

The carrier of $\left(Z, Y, H, Y_{0}\right)$ is $Y_{0} H=\left\{Y_{0} h \mid h \in H\right\}$. But a given $h \in H$ is in some $H_{i}^{\prime}$ and $Y_{0} h$ is in $Y_{0} H_{i}^{\prime}$. But $Y_{0} H_{i}^{\prime}=T_{i}^{\prime}$. This gives $Y_{0} H \subseteq T$. However, each element of $T_{i}^{\prime}$ is clearly in $Y_{0} H$, so $T \subseteq Y_{0} H$.

2.7. Isomorphisms. If $j: Z \rightarrow Z^{\prime}$ is a bijection of sets, then $j$ induces an isomorphism $j_{*}: \operatorname{Sym}(Z) \rightarrow \operatorname{Sym}\left(Z^{\prime}\right)$ by "conjugation" in that $z^{\prime}\left(\sigma j_{*}\right)=z^{\prime}\left(j^{-1} \sigma j\right)$. The quotes can be removed from the word "conjugation" if we put $Z$ and $Z^{\prime}$ in the disjoint union of $Z$ and $Z^{\prime}$.

An isomorphism $f:(Z, Y, H, X) \rightarrow\left(Z^{\prime}, Y^{\prime}, H^{\prime}, X^{\prime}\right)$ of pre-wreath structures is a bijection $f: Z \rightarrow Z^{\prime}$ so that $Y^{\prime}=Y f, X^{\prime}=X f$ and $H^{\prime}=H f_{*}$. We could add the isomorphism $f_{*} \mid H: H \rightarrow H^{\prime}$ to the data of an isomorphism of a pre-wreath isomorphism, but it is not necessary.

Lemma 2.10. (I) If $f:(Z, Y, H, X) \rightarrow\left(Z^{\prime}, Y^{\prime}, H^{\prime}, X^{\prime}\right)$ is an isomorphism of prewreath structures, then $(X H) f=\left(X^{\prime} H^{\prime}\right)$. That is, $f$ takes carrier onto carrier.

(II) If $(Z, Y, H, X)$ is a pre-wreath structure, and $f: Z \rightarrow Z^{\prime}$ is a bijection, then $\left(Z^{\prime}, Y f, H f_{*}, X f\right)$ is a pre-wreath structure and $f:(Z, Y, H, X) \rightarrow\left(Z, Y f, H f_{*}, X f\right)$ is an isomorphism.

2.8. An inductive situation. Let $\left(Z, Y_{1}, H_{1}, Y_{0}\right)$ be a pre-wreath structure, and let $f: Z \rightarrow Z$ be a bijection so that $Y_{0} f=Y_{1}$. Define

$$
\begin{aligned}
Y_{i} & =Y_{0} f^{i}=Y_{1} f^{i-1}, & & i \in \mathbf{Z} \\
H_{i} & =H_{1}\left(f_{*}\right)^{i-1}, & & i \in \mathbf{Z} .
\end{aligned}
$$

This gives that each $\left(Z, Y_{i}, H_{i}, Y_{i-1}\right)$ is a pre-wreath structure for each $i \in \mathbf{Z}$ with carrier $T_{i}=Y_{i-1} H_{i}$. We have $T_{i}=T_{i-1} f=T_{1} f^{i-1}$ for $i \in \mathbf{Z}$.

Recall that $\bar{T}_{i}$ is the union of all the sets in $T_{i}$. We also have $\bar{T}_{i}=\bar{T}_{i-1} f=$ $\bar{T}_{1} f^{i-1}$ for $i \in \mathbf{Z}$. 
We now have a doubly ended sequence of pre-wreath structures and can create various singly ended sequences just by picking different starting places. Thus we have a sequence of singly ended sequences to which Lemma 2.9]applies. We establish notation for the pre-wreath structures that result from applying that lemma.

For $j \in \mathbf{Z}$, let $\left(Z, \widetilde{Y}_{j}, R_{j}, Y_{j-1}\right)$ be the pre-wreath structure that results when Lemma 2.9 is applied to the sequence of pre-wreath structures $\left(Z, Y_{i}, H_{i}, Y_{i-1}\right)$ for $i \geq j$. The notation $R_{j}$ is used since the group is the result of an iteration of wreath products $R_{j}=H_{j} 2 H_{j+1} \prec \cdots$ that goes off to the right. The associativity of the permutation wreath product makes parentheses unnecessary.

It is our intention to make a pre-wreath structure based on the union of the groups $R_{j}$. However we need to make one slight adjustment in the situation so that a carrier of this union is easy to construct.

We know that $\operatorname{Supp}\left(H_{1}\right) \subseteq Y_{1}$. From now on we are going to assume

$$
Y_{0} \subseteq \operatorname{Supp}\left(H_{1}\right) \subseteq Y_{1} \text { and } \operatorname{Supp}\left(H_{1}\right) \neq Y_{1} .
$$

Since $Y_{0} \subseteq \operatorname{Supp}\left(H_{1}\right)$ and $Y_{0} f=Y_{1}$, we have a non-empty subset $W_{0}$ of $Y_{0}$ so that $W_{0} f \cap \operatorname{Supp}\left(H_{1}\right)=\emptyset$. Thus $W_{0} f \subseteq\left(Y_{1} \backslash \operatorname{Supp}\left(H_{1}\right)\right)$. If we define $W_{i}=W_{0} f^{i}$ for $i \in \mathbf{Z}$, then it follows from $Y_{i}=Y_{i-1} f$ and $\operatorname{Supp}\left(H_{i}\right)=\operatorname{Supp}\left(H_{i-1}\right) f$ for all $i \in \mathbf{Z}$ that $W_{i} \subseteq\left(Y_{i} \backslash \operatorname{Supp}\left(H_{i}\right)\right)$. The following picture might help.

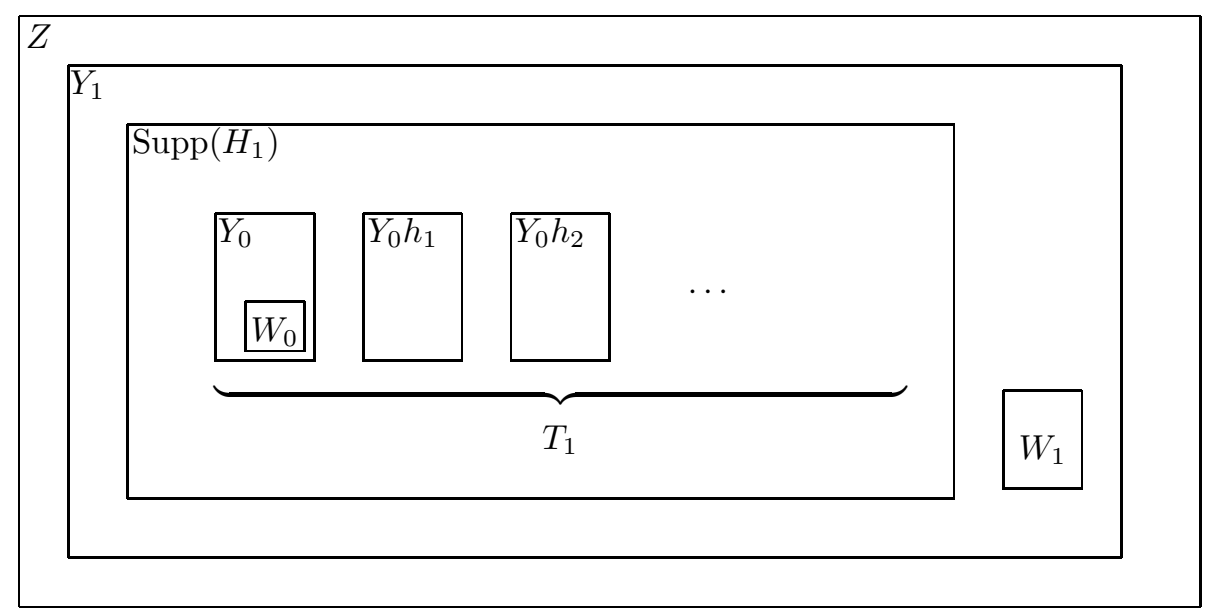

A trivial conclusion from the fact that $H_{1}$ is not trivial and $Y_{0}$ is not empty is that $\operatorname{Supp}\left(H_{1}\right)$ is not empty and contains all of $Y_{0} H_{1}$. In turn, the non-empty $\operatorname{Supp}\left(H_{1}\right)$ is contained in $Y_{1}=Y_{0} f$ which is contained in $\operatorname{Supp}\left(H_{2}\right)=\left(\operatorname{Supp}\left(H_{1}\right)\right) f$. Eventually, $\operatorname{Supp}\left(H_{i}\right) \subseteq \operatorname{Supp}\left(H_{j}\right)$ for all $i<j$.

Lemma 2.11. Let $\left(Z, Y_{1}, H_{1}, Y_{0}\right)$ be a pre-wreath structure, and let $f: Z \rightarrow Z$ be a bijection so that $Y_{0} f=Y_{1}$. Define $Y_{i}, H_{i}, T_{i}, \widetilde{Y}_{j}$, and $R_{j}$ as above. Assume that (6) holds, let $\emptyset \neq W_{0} \subseteq Y_{0}$ so that $W_{0} f \cap \operatorname{Supp}\left(H_{1}\right)=\emptyset$, and let $W_{i}=W_{0} f^{i}$ for $i \in \mathbf{Z}$. Then for each $j \in \mathbf{Z}$, the following are true.

(a) $\left(Z, \tilde{Y}_{j}, R_{j}, Y_{j-1}\right)$ and $\left(Z, \tilde{Y}_{j}, R_{j}, W_{j-1}\right)$ are pre-wreath structures.

(b) $f:\left(Z, \widetilde{Y}_{j}, R_{j}, Y_{j-1}\right) \rightarrow\left(Z, \widetilde{Y}_{j+1}, R_{j+1}, Y_{j}\right)$ and $f:\left(Z, \widetilde{Y}_{j}, R_{j}, W_{j-1}\right) \rightarrow$ $\left(Z, \widetilde{Y}_{j+1}, R_{j+1}, W_{j}\right)$ are isomorphisms.

(c) $R_{j} \subseteq R_{j+1}$. 
(d) The various carriers are related by $W_{j-1} R_{j} \subseteq Y_{j-1} R_{j} \subseteq Y_{j} R_{j+1}$, and $\overline{Y_{j-1} R_{j}} \cap \overline{W_{j} R_{j+1}}=\emptyset$.

(e) For all $k \neq j$, the underlying sets $\overline{W_{j-1} R_{j}}$ and $\overline{W_{k-1} R_{k}}$ are disjoint.

(f) For $j<k$ the sets in $W_{k-1} R_{k}$ are disjoint from $\operatorname{Supp}\left(H_{j}\right)$.

Proof. Item (a) follows from Lemmas 2.9 and 2.3 The verifications for (b) and (c) are elementary. For (d), the containments follow from $W_{0} \subseteq Y_{0} \subseteq Y_{1}$ and the properties of the action of $f$ and its powers. If we show that $\overline{Y_{0} R_{1}} \cap \overline{W_{1} R_{2}}=\emptyset$, then the disjointness claim in (d) will then follow by applying powers of $f$.

By Lemma 2.9.

$$
Y_{0} R_{1}=\bigcup_{i \geq 1} Y_{0} H_{1} H_{2} \cdots H_{i}=\bigcup_{i \geq 2} T_{1} H_{2} H_{3} \cdots H_{i} .
$$

A similar formula for $Y_{1} R_{2}$ and Definition 2.1(4) gives

$$
W_{1} R_{2}=\bigcup_{i \geq 2} W_{1} H_{2} H_{3} \cdots H_{i}=\bigcup_{i \geq 2} W_{0} f H_{2} H_{3} \cdots H_{i} .
$$

It suffices to show $\overline{W_{0} f H_{2} H_{3} \cdots H_{i}} \cap \overline{T_{1} H_{2} H_{3} \cdots H_{i}}=\emptyset$ for each $i \geq 2$.

We already know that $W_{0} f \cap \bar{T}_{1}=\emptyset$. Both $W_{0} f H_{2} H_{3} \cdots H_{i}$ and $\bar{T}_{1} H_{2} H_{3} \cdots H_{i}$ are collections of subsets of $Y_{i}$. If we have disjoint subsets $A$ and $B$ of $Y_{i}$, then we are done if we show that $A H_{i+1}$ and $B H_{i+1}$ are disjoint. But if they are not, then $A h \cap B h^{\prime} \neq \emptyset$ for some $h$ and $h^{\prime}$ in $H_{i+1}$, so that $Y_{i} h \cap Y_{i} h^{\prime} \neq \emptyset$. This implies that $\left.h\right|_{Y_{i}}=\left.h^{\prime}\right|_{Y_{i}}$ which is impossible if the disjoint $A$ and $B$ in $Y_{i}$ have intersecting images under $h$ and $h^{\prime}$.

Item (e) follows from the truth of (d) for all $j \in \mathbf{Z}$.

Lastly, $\operatorname{Supp}\left(H_{k-1}\right)$ is disjoint from $W_{k-1}$ and both are in $Y_{k-1}$. By (4) of Definition 2.1] every element of $R_{k}$ that takes points of $Y_{k-1}$ into $Y_{k-1}$ is the identity on $Y_{k-1}$. Thus $\operatorname{Supp}\left(H_{k-1}\right)$ is disjoint from all sets in $W_{k-1} R_{k}$. Now every $\operatorname{Supp}\left(H_{j}\right)$ is contained in $\operatorname{Supp}\left(H_{k-1}\right)$ for $j<k$.

Lemma 2.12. Assume the hypotheses and notation of Lemma[2.11, Let $L_{i}$ be the group $\left\langle H_{i-1}, H_{i-2}, \ldots\right\rangle$, and let $M$ be the union of all the $R_{i}$. Then $M$ is also the union of all the $L_{i}$ and is also generated by the union of all the $H_{i}$. Further, for each $i \in \mathbf{Z}$, the following are true.

(a) $f: L_{i} \rightarrow L_{i+1}$ is an isomorphism.

(b) $M=L_{i} \curlywedge R_{i}$.

(c) For each $k>0, L_{i+k}=L_{i} \imath\left\langle H_{i}, H_{i+1}, \ldots, H_{i+k-1}\right\rangle$.

(d) Each non-trivial normal subgroup of $L_{i}$ and each non-trivial normal subgroup of $M$ contains a subgroup that surjects onto $L_{0}$.

Proof. That statements about what constitutes $M$ are immediate. Item (a) is immediate. Item (b) follows from the fact that all of the $H_{j}$ are represented in $L_{i} \cup R_{i}$, the fact that $\left(Z, \widetilde{Y}_{i}, R_{i}, Y_{i-1}\right)$ is a pre-wreath structure, the fact that the support of $L_{i}$ is in $Y_{i-1}$, and from Lemma 2.6 Item (c) is similar. To argue (d), we note that a non-trivial normal subgroup $N$ of $L_{i}$ or $M$ contains an element that moves a set in some $W_{j-1} R_{j}$. Now by Lemma 2.8 applied to $M=L_{j} \prec R_{j}$ or $L_{i}=L_{j} \imath\left\langle H_{j+1}, \ldots, H_{i-1}\right\rangle$, a subgroup of $N$ surjects onto $L_{j}$ which is isomorphic to $L_{0}$.

Note that we do not claim a pre-wreath structure for $M$ or for any of the $L_{i}$. 
Proposition 2.13. Assume the hypotheses and notation of Lemma 2.11 and let

$$
V=\langle M, f\rangle=\left\langle L_{1}, f\right\rangle=\left\langle R_{1}, f\right\rangle=\left\langle H_{1}, f\right\rangle .
$$

Then $V$ is the ascending $H N N$ extension of $R_{1}$ given by the injection of $R_{1}$ in itself under conjugation by $f$ and it is also the semidirect product of $M$ with the infinite cyclic group generated by $f$. Further, $\left(Z, Y_{1} \cup \operatorname{Supp}(f), V, W_{0}\right)$ is a prewreath structure whose carrier $W_{0} V$ is the union of the $W_{0} R_{1} f^{n}, n \in \mathbf{Z}$. Lastly, any non-trivial normal subgroup of $V$ contains a subgroup that surjects onto $L_{0}$.

Proof. The claimed equalities are immediate and the claimed structures as HNN extensions and semidirect products follow from the standard characterizations of those structures.

We look at the five items in Definition 2.1 of a pre-wreath structure. Item (1) is immediate. Item (2) follows from the fact that the support of $H_{1}$ is in $Y_{1}$. Item (3) is immediate.

To work on the remaining items and the claim about $W_{0} V$, we consider an arbitrary element $v$ of $V$. From Lemma 2.4 $v=f^{n} h_{1} h_{2} h_{3} \cdots h_{k}$ where each $h_{i}$ is in a conjugate of $H_{1}$ by a power of $f$. Thus for each $i$ there is a $j_{i}$ so that $h_{i} \in H_{j_{i}}$. Write $v$ as $v=f^{n} w$ with $w=h_{1} h_{2} h_{3} \cdots h_{k}$. Writing $M=L_{n+1} \prec R_{n+1}$, let $\phi_{n}: M \rightarrow R_{n+1}$ be as given by Lemma 2.7 and let $\bar{w}$ be the image of $w$ under $\phi_{n}$. This is just $w$ with all $h_{i}$ removed that have $j_{i} \leq n$.

Now $W_{0} v=W_{0} f^{n} w$ and $\left.w\right|_{W_{n}}=\left.\bar{w}\right|_{W_{n}}$ since every factor of $\bar{w}$ is in $R_{n+1}$ and by Lemma 2.11 (f), all sets in $W_{n} R_{n+1}$ are disjoint from the support of $L_{n+1}$. This puts $W_{0} v$ in $W_{n} R_{n+1}=W_{0} R_{1} f^{n}$. That $W_{0} V$ contains all the $W_{n} R_{n+1}$ is clear.

We consider (4). If $W_{0} v$ intersects $W_{0}$, then by Lemma 2.11(e) and the above argument, we must have that the form of $v$ is as $w$ above: a product of elements of the various $H_{i}$. Now let $\bar{w}$ be the image of $w$ under $\phi_{0}: M \rightarrow R_{1}$. Our argument above has $\left.v\right|_{W_{0}}=\left.\bar{w}\right|_{W_{0}}$. But $\bar{w}$ is in $R_{1}$ and $\left(Z, \widetilde{Y}_{1}, R_{1}, W_{0}\right)$ is a prewreath structure, so $\left.\bar{w}\right|_{W_{0}}=\left.1\right|_{W_{0}}$.

To work on (5), we start with a $v \in V$. If $v=f^{n} w$ with $w$ a product of elements in the $H_{i}$ and $n \neq 0$, then $W_{0} v$ is in $W_{n} R_{n+1}$ by our analysis above. By Lemma 2.11(e), $W_{0}$ is not in $W_{n} R_{n+1}$ so $v$ moves $W_{0}$. If $n=0$, then $v$ is in some $R_{k}$. We can take $k$ to be the smallest $i$ so that $H_{i}$ contains a factor of $w$. Now $v$ must move some element $X$ of $W_{i-1} R_{i}$. But $X$ is of the form $W_{0} v^{\prime}$ for some $v^{\prime} \in V$.

For the final claim, let $N$ be a non-trivial normal subgroup of $V$. If $N \subseteq M$, then Lemma 2.12 (c) gives the conclusion. If not, then an element of $N$ is of the form $v=f^{n} w$ as above with $n \neq 0$ and with $w$ in some $L_{i}$. Now if $h$ is in $H_{i-n}$, then

$$
\begin{aligned}
c=v^{-1} h v h^{-1} & =w^{-1} f^{-n} h f^{n} w h^{-1} \\
& =w^{-1}(h)^{f^{n}} w h^{-1}
\end{aligned}
$$

is in $M \cap N$ and the calculation shows that $c \phi_{n-1}$ is non-trivial no matter whether $n$ is positive or negative, where $\phi_{n-1}: M \rightarrow R_{n}$ is the epimorphism from Lemma 2.7 Now Lemma 2.12 (c) applies once more.

2.9. Remarks. There is very little in this section that is new. As mentioned in 22], wreath products are often used to supply examples of groups with special properties. In 23], they are used to build groups in which others embed. By contrast, more of our effort goes towards recognition than creation, and so our view 
of the wreath product is more "internal" than "external." However this shift of view contains no surprises.

The reference [28] that we give for the wreath product of permutation groups is not the oldest and the notion seems to have been introduced in 24]. Iterated wreath products abound. The group we call $M$ in Lemma 2.12 is the group $W$ of (5) on Page 171 of [21]. The fact that groups of homeomorphisms of the reals interact strongly with wreath products is in 22] and 23] with the latter having more overlap with the current paper. The remarks at the beginning of Section 5 of 23] relate to the effort that we put into finding "carriers" of the various groups.

\section{Elementary amenability Classes}

3.1. Squares and higher products. We start with some elementary observations on elementary classes. Since $E G_{0}$ contains both finite groups (which might not be abelian) and abelian groups (which might not be finite), it is clear that $G \times H$ might not be in $E G_{0}$ even if both $G$ and $H$ are in $E G_{0}$. However, $G \times G$ will always be in $E G_{0}$ if $G$ is in $E G_{0}$. The following can be shown as an exercise.

Lemma 3.1. If $G$ is in $E G_{\alpha}$, then $G \times G$ is also in $E G_{\alpha}$.

On the other hand, if $G \in E G_{0}$ is finite, then $\Sigma(G)$, the direct sum of countably many copies of $G$ (all sequences in $G$ that are eventually the identity under pointwise multiplication) is not in $E G_{0}$. However, if $G \in E G_{0}$ is abelian, then $\Sigma(G)$ is in $E G_{0}$. This behavior is important to us and we make it a definition.

Definition 3.2. If $G \in E G$ has elementary class $\alpha$, then we say that $G$ has property $\Sigma$ if $\Sigma(G)$ also has elementary class $\alpha$.

3.2. Property $\Sigma$ and wreath products. There are groups of class 0 with property $\Sigma$ because of the abelian groups. We get more from the next lemma.

Lemma 3.3. If $(G, Z)$ is a transitive, countably infinite, finitely generated permutation group so that $G$ is in $E G$ with elementary class $\alpha$ and with property $\Sigma$, then $G \imath G$ has elementary class $\alpha+1$ and has property $\Sigma$.

Proof. Non-trivial wreath products are not abelian and our hypotheses imply that

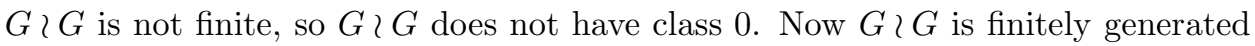
and any representation of $G \imath G$ is a union of groups must have $G \imath G$ as one of the groups. If $G$ < $G$ is the middle term of a short exact sequence, then we can apply Lemma 2.8 since there is no problem realizing $G \succ G$ as a result of Proposition 2.5 Now Lemma 2.8 and Proposition 1.1] say that either the kernel or quotient in the short exact sequence has class at least that of $G$. Thus the class of $G$ ? $G$ is at least $\alpha+1$. However, by the structure of a wreath product and the countability of $G$, there is a normal subgroup $N$ of $G$ isomorphic to $\Sigma(G)$ whose corresponding quotient is $G$. By hypothesis, the class of $\Sigma(G)$ is $\alpha$, so the class of $G \_G$ is no more than $\alpha+1$.

It is not hard to represent a countable direct sum $H$ of copies of $G\{G$ as a wreath product. The top group of $H$ will be a copy of $\Sigma(G)$. The base group of $H$ is a countable direct sum of copies of the base group $N$ in $G$ ? $G$. Thus the base group of $H$ is isomorphic to $\Sigma(G)$, and we get that $H$ ends up with class $\alpha+1$ as well.

We make the next lemma more specific to the constructions in Section 2 
Lemma 3.4. Let $\alpha$ be an ordinal and let $\beta$ be the smallest limit ordinal that is greater than $\alpha$. Assume the notation and hypotheses of Proposition 2.13 and assume that $\left(H_{1}, Y_{0} H_{1}\right)$ is a transitive, countably infinite, finitely generated permutation group so that $H_{1}$ is in $E G$ with elementary class $\alpha$ and with property $\Sigma$. Then the elementary class of $M$ is $\beta+1$, the elementary class of $V$ is $\beta+2$ and both $M$ and $V$ have property $\Sigma$.

Proof. All the $H_{i}$ are isomorphic and the normal subgroup $M$ of Proposition 2.13 contains groups that can be written as arbitrarily long sequences of the form $H_{1} 2$ $H_{1} \imath \cdots \imath H_{1}$. Thus $M$ contains all subgroups in the inductively defined sequence $P_{0}=H_{1}, P_{i+1}=P_{i} \prec P_{i}$. By Lemma 3.3 $M$ contains subgroups of elementary class $\alpha+i$ for all $i \in \mathbf{N}$. Thus $M$ is in none of the $E G_{\alpha+i}$ and is thus not in $E G_{\beta}$ which is the union of all the $E G_{\alpha+i}$ by definition. However, $M$ is the union of the $P_{i}$ and so is in $E G_{\beta+1}$ and the elementary class of $M$ is $\beta+1$.

Since $V=\left\langle H_{1}, f\right\rangle$, it is finitely generated and getting $V$ as a union of groups of lower class is ruled out. Since any non-trivial normal subgroup of $V$ contains a copy of $L_{0}$ which in turn contains a copy of every $P_{i}$, the class of $V$ is at least $\beta+2$. However, $V$ is the semidirect product of $M$ and a copy of the integers, so the class of $V$ is no more than $\beta+2$.

Each $P_{i}$ has property $\Sigma$ and $\Sigma(M)$ is the union of the $\Sigma\left(P_{i}\right)$, so $M$ has property $\Sigma$. Now $\Sigma(\mathbf{Z})$ has class 0 , and $\Sigma(V)$ is the semidirect product of $\Sigma(M)$ with $\Sigma(\mathbf{Z})$. If follows that the class of $\Sigma(V)$ is that of $V$.

The next is an easy exercise from the definitions of the $E G_{\alpha}$.

Lemma 3.5. If $G_{i}$ is a sequence of groups in $E G$ whose sequence of elementary classes has no largest element, and beta is the limit of the classes, then the direct sum of the $G_{i}$ has elementary class $\beta+1$.

\section{THE MAIN THEOREM}

4.1. $F$ or $P L_{o}(I)$. In this section we will build subgroups of $F$, but we will spend little time talking about $F$. Our groups will be groups of homeomorphisms from the unit interval to itself and we will build the groups by picking out homeomorphisms. Technically, $F$ is the subgroup of $P L_{o}(I)$ in which all slopes used are integral powers of two, and all discontinuities of slope occur at points in $\mathbf{Z}\left[\frac{1}{2}\right]$, the set of rational numbers whose denominators are integral powers of two [8, Section 1]. Thus, we should appear to be careful and only use homeomorphisms possessed of the properties of homeomorphisms in $F$. However, we will not do so.

There is incredible flexibility possessed by elements of $F$ that will allow us to assume (without explicitly saying so beyond this paragraph) that all that we build can be built in $F$. The key fact is the following. Given any two finite sequences $x_{1}<x_{2}<\cdots<x_{k}$ and $y_{1}<y_{2}<\cdots<y_{k}$ of the same length in $\mathbf{Z}\left[\frac{1}{2}\right] \cap[0,1]$, there is an element of $F$ taking each $x_{i}$ to $y_{i}$. In particular, if $x_{1}=y_{1}$ and $x_{k}=y_{k}$, then the element of $F$ can be chosen to be the identity outside of the interval $\left[x_{1}, x_{k}\right]$. This is recorded in several places, such as Sections 1 and 2.2 of [3]. From this point on, we will assume that any element called for with certain properties in $P L_{o}(I)$ can be chosen to come from $F$.

4.2. Bumps. We adapt terminology from ordered groups. All functions discussed here are elements of $H_{o}(I)$, the group of orientation preserving self homeomorphisms of the unit interval $I=[0,1]$. 
If $h \in H_{o}(I)$, then a bump interval of $h$ is an interval $[a, b]$ in $I$ with $a<b$ so that $a$ and $b$ are fixed points of $h$ and no $x \in(a, b)$ is fixed by $h$. That is, a bump interval of $h$ is a maximal closed interval whose non-empty interior contains no fixed points of $h$. A one bump function is a function that has only one bump interval. Two examples of one bump functions are pictured below.
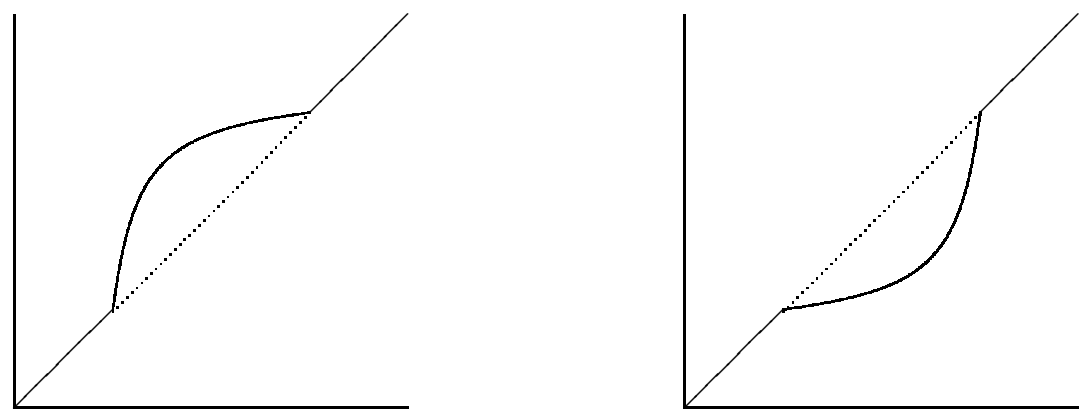

The word "bump" by itself will be dealt with less precisely. If $h$ is in $H_{o}(I)$, then a bump of $h$ is either the restriction of $h$ to one of its bump intervals, or it is the unique one bump function that agrees with $h$ on a given bump interval. However, we will sometimes be careless and use the word bump to refer to a bump interval, if at the same time it is also being used to refer to a function on that interval. This allows us to make such statements as "disjoint bumps commute." We hope the reader is comfortable with this.

Given a one bump function $h$ with bump interval $[a, b]$ a fundamental domain for $h$ is an interval $[c, d]$ in $(a, b)$ with $c<d$ so that $c h=d$ or $d h=c$. Examples are pictured below.
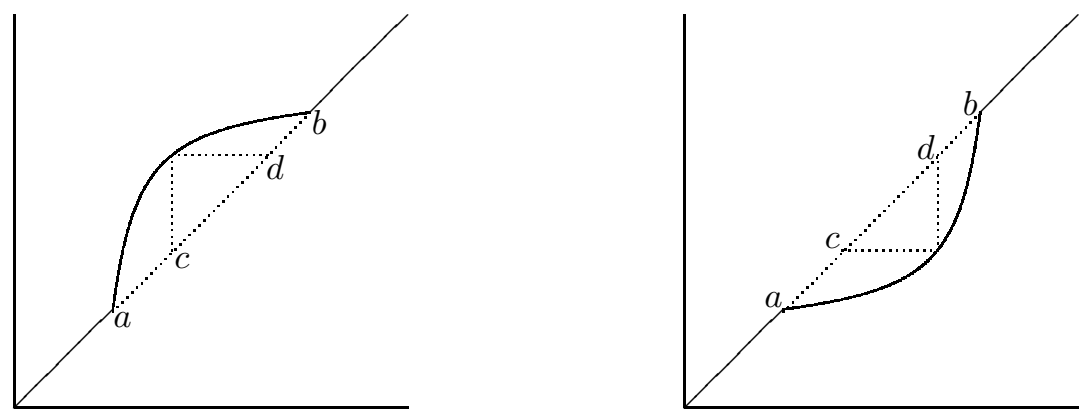

The extra generality in the next lemma is deliberate.

Lemma 4.1. If $I_{i}, i=1,2,3,4$, are intervals in $I=[0,1]$ with $I_{i}$ contained in the interior of $I_{i+1}$, so that $h$ is a one bump function with bump interval $I_{3}$ and fundamental domain $I_{2}$ then $\left(I, I_{4},\langle h\rangle, I_{1}\right)$ is a pre-wreath structure.

4.3. Finding room. There is a lot of room in the unit interval. We will not say a lot about where to put the elements and groups that we create. Room can always be found and we will leave it to the reader to find the room. A key tool in finding the room is the next standard lemma. 
Lemma 4.2. Any countable ordinal $\alpha$ can be embedded in an order preserving way in $I=[0,1]$.

Proof. Let $f$ be a bijection from $\alpha$ to the set

$$
\left\{I_{i}=\left[\frac{1}{2^{i+1}}, \frac{1}{2^{i}}\right] \mid i \in \mathbf{N}\right\} .
$$

The embedding sends $\beta \in \alpha$ to the measure of the set $\bigcup_{\gamma<\beta} f(\gamma)$, and elementary measure theory establishes the needed properties of the embedding.

4.4. The constructions. We start with the group generated by a one bump function. This is isomorphic to $\mathbf{Z}$, has elementary class 0 and has property $\Sigma$. This can be put in a pre-wreath setting by Lemma 4.1

The inductive step is to take a finitely generated group of class $\alpha$ with property $\Sigma$ in a pre-wreath structure $(I, J, G, K)$ where $J$ and $K$ are intervals in the interior of $I$ and where the support of $G$ has closure in the interior of $J$. Then a function $f$ can be found that takes $K$ to $J$. We can further ask that the support of $f$ have closure in the interior of $I$. Now Lemmas 2.11 2.12 and Proposition 2.13 apply as well as Lemmas 3.3 and 3.4 to give groups with classes $\alpha+i$ for $i \in \mathbf{N}$ and $\beta+1$ and $\beta+2$ where $\beta$ is the limit of the $\alpha+i$. If we call the largest group $V(G)$, then we also get a pre-wreath structure for $V(G)$. The group $V(G)$ will be finitely generated as well. This process can then be repeated.

If we start with $G_{0}$ as the group generated by a one-bump function, then we can create $G_{i+1}$ as $V\left(G_{i}\right)$. In this way we get groups of all classes except limit classes from class 0 up to but not including the class $\omega^{2}$. For the class $\omega^{2}+1$, we take the direct sum of the $G_{i}$ and apply Lemma 3.5 The direct sum is obtained by finding a countable set of pairwise disjoint closed intervals in $I$ and conjugating each $G_{i}$ into its own private interval. The group generated by the images of the $G_{i}$ is the desired group.

We have shown Theorem 1 .

4.5. Going farther. If we let $\widetilde{G}$ be the direct sum of the $G_{i}$ of the previous section, then $\widetilde{G}$ is not finitely generated. Various constructions on $\widetilde{G}$ do not seem to raise the class. It is not hard to see that $\widetilde{G} \imath \widetilde{G}$ and $V(\widetilde{G})$ have the same class as $\widetilde{G}$.

There remains the possibility that there are other construction techniques to try. A few attempts at other constructions gave groups not in the class EG. In particular, they gave groups that had subgroups isomorphic to the non-elementary amenable group $F$. The presence of the group $F$ is not hard to detect using the main result of [2].

\section{TWO NON-SOLVABLE GROUPS}

In this section we consider the two groups

$$
\begin{aligned}
& (\mathbf{Z} \imath)^{\infty}=\mathbf{Z} \imath \mathbf{Z} \imath \mathbf{Z} \imath \cdots, \\
& \infty(\imath \mathbf{Z})=\cdots \text { ไ } \mathbf{Z} \text { ? } \mathbf{Z} \text { ? } \mathbf{Z},
\end{aligned}
$$

where parentheses have been left out because the wreath products are all wreath products of permutation groups.

These groups are subgroups of the group $G_{1}$ of the previous section. The group $G_{1}$ can be viewed as generated by the functions $h$ and $f$ in the diagram below. We 
show smooth bumps, but the reader should interpret the pictures as representing PL functions (or more restrictively, elements of $F$ ).

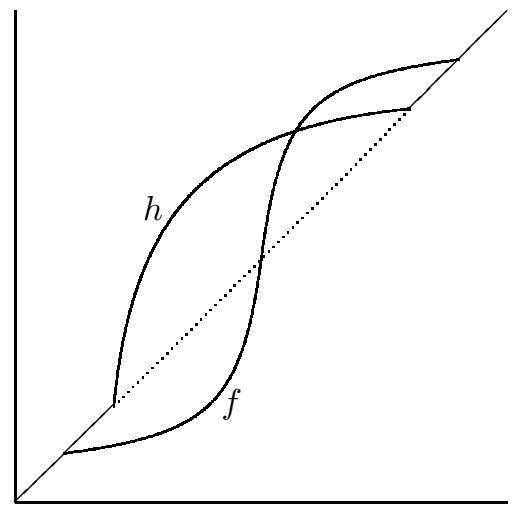

The next picture shows how $f^{-1}$ conjugates $h$ into a one bump function whose one bump interval fits properly inside a fundamental domain of $h$.

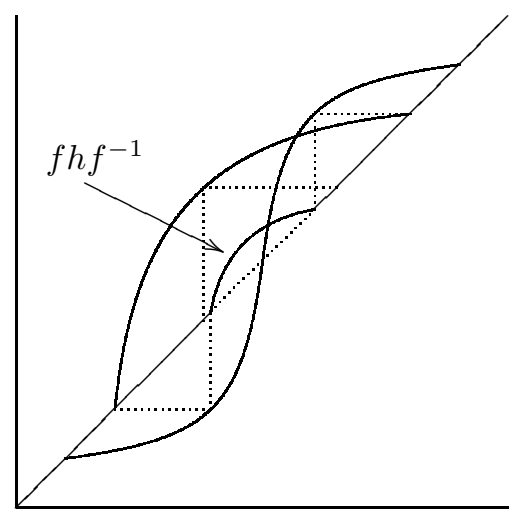

If we let $h_{i}=f^{-i} h f^{i}$ for $i \in \mathbf{Z}$, then $h=h_{0}$ and the function $f h f^{-1}$ shown is $h_{-1}$. It is easier to show than $h_{1}$.

The group $(\mathbf{Z} \imath)^{\infty}$ is generated by $h_{1}, h_{2}, \ldots$ while $h_{-1}, h_{-2}, \ldots$ generates $\infty(\imath \mathbf{Z})$.

In view of the fact that we already know that $(\mathbf{Z} \imath)^{\infty}$ and ${ }^{\infty}(\imath \mathbf{Z})$ embed in $F$ and $P L_{o}(I)$, Theorem 2 will follow from the next two lemmas. We start with the easier of the two.

Lemma 5.1. There is no subgroup of $(\mathbf{Z} \imath)^{\infty}$ that is isomorphic to ${ }^{\infty}(\imath \mathbf{Z})$.

Proof. The subgroups $A_{i}=\left\langle h_{1}, h_{2}, \ldots, h_{i}\right\rangle$ exhaust $(\mathbf{Z} \imath)^{\infty}$. If we define the groups $B_{i}=\left\langle h_{i+1}, h_{i+2}, \ldots\right\rangle$, then we have $(\mathbf{Z} \imath)^{\infty}=A_{i}\left\langle B_{i}\right.$. The kernel $K_{i}$ of the surjection $(\mathbf{Z} \imath)^{\infty} \rightarrow B_{i}$ of Lemma 2.7 is isomorphic to $\Sigma\left(A_{i}\right)$, the countable direct sum of copies of $A_{i}$. But $A_{i}$ has class no greater than $i$ (it is closer to $\log _{2}(i)$ ) and $A_{i}$ has property $\Sigma$ (exercise). Thus the class of each $K_{i}$ is finite, and the $K_{i}$ exhaust $(\mathbf{Z} \text { ? })^{\infty}$.

If the lemma is false, then the intersections of the $K_{i}$ with the embedded ${ }^{\infty}(2 \mathbf{Z})$ will exhaust $\infty(\zeta \mathbf{Z})$ with normal subgroups. But from Lemma2.12 every non-trivial normal subgroup of $\infty(\zeta \mathbf{Z})$ contains a subgroup that surjects onto $\infty(\zeta \mathbf{Z})$ and so has class at least that of ${ }^{\infty}(\imath \mathbf{Z})$. But ${ }^{\infty}(\imath \mathbf{Z})$ contains arbitrarily long $\left.\mathbf{Z} \imath \mathbf{Z} \imath \cdots\right\urcorner \mathbf{Z}$ 
iterations of wreath products of $\mathbf{Z}$ and so does not have finite class. However, this would have to be a subgroup of some $K_{i}$ with its finite class - a contradiction.

Lemma 5.2. There is no subgroup of ${ }^{\infty}(2 \mathbf{Z})$ that is isomorphic to $(\mathbf{Z} \imath)^{\infty}$.

The following proof can be reworded in terms of the action of $\infty(2 \mathbf{Z})$ on a rooted tree of infinitely many levels in which each node has a $\mathbf{Z}$ indexed set of children. We did not feel that it was worth making the translation.

Proof. Assume a monomorphism from $(\mathbf{Z} \imath)^{\infty}$ into ${ }^{\infty}(\zeta \mathbf{Z})$. For an element $x \in$ $(\mathbf{Z} \text { ? })^{\infty}$, we will write its image as $\bar{x}$.

The group $(\mathbf{Z} \imath)^{\infty}$ is generated by $h_{1}, h_{2}, \ldots$ which satisfy the following whenever $1 \leq i<j<k$ :

$$
\left[h_{i},\left(h_{k}\right)^{-n}\left(h_{j}\right)\left(h_{k}\right)^{n}\right]=1 \text { if and only if } n \neq 0 .
$$

In words, $h_{j}$ is the only conjugate of $h_{j}$ by a power of $h_{k}$ that fails to commute with $h_{i}$.

We will focus on the fact that (圂) must be satisfied by the $\bar{h}_{1}, \bar{h}_{2}, \ldots$ in ${ }^{\infty}(2 \mathbf{Z})$.

We will talk a great deal about bumps.

Elements of ${ }^{\infty}(\imath \mathbf{Z})$ are made of finitely many pairwise disjoint bumps. Given two bumps from different elements of $\infty(2 \mathbf{Z})$, the intervals are either disjoint, identical, or one is in the interior of the other. This is not hard to show as an exercise (which can further show that bump intervals in $\infty(2 \mathbf{Z})$ are very restricted in that if the left endpoint of a bump is known, then its right endpoint is determined and vice versa).

If two bumps are related by having the interval of one in the interior of the interval of the other, then we will say that they are "nested." The bump with the larger interval will be called "superior to" the bump with the smaller interval and the inverse relation will be called "inferior to."

It is extremely important that an inferior bump in $\infty(2 \mathbf{Z})$ is always contained in a fundamental domain of a superior bump.

Nested bumps cannot commute.

Another important fact that we need is that in $\infty(2 \mathbf{Z})$, any bump interval is inferior to only finitely many other bump intervals from ${ }^{\infty}(2 \mathbf{Z})$.

We need a sublemma: If $u, v$ and $w$ are bumps of piecewise linear functions on the same bump interval $[a, b]$ and if $w^{-m} v w^{m}$ and $w^{-n} v w$ both commute with $u$, then $w^{n-m}$ commutes with $v$.

Proof of the sublemma. This is the only argument in the paper that uses the PL setting. A function with bump interval $[a, b]$ that commutes with $u$ is completely determined by what it does on a fundamental domain of $u$ in $[a, b]$. There are infinitely many fundamental domains of $u$ in any $[a, a+\epsilon)$ with $\epsilon>0$. Because the functions are piecewise linear and $a$ is a fixed point of $v$ and $w$, there is an $[a, a+\epsilon)$ on which all of $v, w^{-m} v w^{m}$ and $w^{-n} v w^{n}$ agree. Since the last two commute with $u$, they are identical and the sublemma is proved.

Proof of Lemma 5.2, continued. We first argue that if there are $i<j$ so that $\bar{h}_{i}$ and $\bar{h}_{j}$ have nested bumps, then we have a contradiction. From (図), we know that $\bar{h}_{j+1}$ must conjugate $\bar{h}_{j}$ so that no bumps from $\bar{h}_{i}$ and the conjugate of $\bar{h}_{j}$ are nested. This can only happen if a bump of $\bar{h}_{j+1}$ is superior to the relevant bumps of both $\bar{h}_{i}$ and $\bar{h}_{j}$. Now the argument can be repeated for $\bar{h}_{j}$ and $\bar{h}_{j+1}$ since they have 
nested bumps. In this way we create an infinite ascending chain of nested bumps. As observed above, this is not possible in $\infty(2 \mathbf{Z})$.

We now assume that for all $i \neq j$, if bump intervals of $\bar{h}_{i}$ and $\bar{h}_{j}$ intersect, then they are identical. Since $\bar{h}_{1}$ and $\bar{h}_{2}$ do not commute, there has to be at least one interval $[a, b]$ that is a bump interval for bumps of $\bar{h}_{1}$ and $\bar{h}_{2}$ that do not commute. By (困), all non-zero powers of $\bar{h}_{3}$ conjugate $\bar{h}_{2}$ so that the results commute with $\bar{h}_{1}$. By our latest assumption, this is accomplished by a bump of $\bar{h}_{3}$ whose interval is also $[a, b]$. By the sublemma, $\bar{h}_{3}$ commutes with $\bar{h}_{2}$ on $[a, b]$. But this makes it impossible for $\bar{h}_{3}$ to alter $\bar{h}_{2}$ on $[a, b]$ by conjugation. Since $\bar{h}_{2}$ does not commute

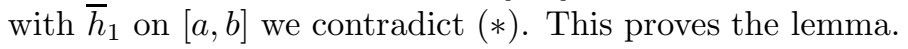

\section{REFERENCES}

1. S. I. Adyan, Random walks on free periodic groups, Izv. Akad. Nauk SSSR Ser. Mat. 46 (1982), no. 6, 1139-1149, 1343. MR 84m:43001

2. Matthew G. Brin, The ubiquity of Thompson's group $F$ in groups of piecewise linear homeomorphisms of the unit interval, J. London Math. Soc. (2) 60 (1999), no. 2, 449-460. MR 1 724861

3. Matthew G. Brin and Fernando Guzmán, Automorphisms of generalized Thompson groups, J. Algebra 203 (1998), no. 1, 285-348.

4. Matthew G. Brin and Craig C. Squier, Groups of piecewise linear homeomorphisms of the real line, Invent. Math. 79 (1985), 485-498.

5. Kenneth S. Brown, Finiteness properties of groups, Journal of Pure and Applied Algebra 44 (1987), 45-75.

6. J. Burillo, S. Cleary, and M. I. Stein, Metrics and embeddings of generalizations of Thompson's group F, Trans. Amer. Math. Soc. 353 (2001), no. 4, 1677-1689 (electronic). MR 2001k:20087

7. José Burillo, Quasi-isometrically embedded subgroups of Thompson's group F, J. Algebra 212 (1999), no. 1, 65-78.

8. J. W. Cannon, W. J. Floyd, and W. R. Parry, Introductory notes on Richard Thompson's groups, Enseign. Math. (2) 42 (1996), no. 3-4, 215-256. MR 98g:20058

9. Ching Chou, Elementary amenable groups, Illinois J. Math. 24 (1980), no. 3, 396-407. MR 81h:43004

10. Sean Cleary and Jennifer Taback, Geometric quasi-isometric embeddings into Thompson's group F, New York J. Math. 9 (2003), 141-148 (electronic). MR 2016187

11. Mahlon M. Day, Amenable semigroups, Illinois J. Math. 1 (1957), 509-544. MR 19,1067c

12. Patrick Dehornoy, The structure group for the associativity identity, J. Pure Appl. Algebra 111 (1996), no. 1-3, 59-82. MR 97d:55033

13. J. Dydak, 1-movable continua need not be pointed 1-movable, Bull. Acad. Polon. Sci. Sér. Sci. Math. Astronom. Phys. 25 (1977), 485-488.

14. - A simple proof that pointed, connected FANR-spaces are regular fundamental retracts of ANR's, Bull. Acad. Polon. Sci. Sér. Sci. Math. Astronom. Phys. 25 (1977), 55-62.

15. Peter Freyd and Alex Heller, Splitting homotopy idempotents II, Journal of Pure and Applied Algebra 89 (1993), 93-106.

16. S. M. Gersten, Selected problems, Combinatorial Group Theory and Topology (S. M. Gersten and John R. Stallings, eds.), Annals of Mathematics Studies, vol. 111, Princeton University Press, 1987, pp. 545-551.

17. R. I. Grigorchuk, Degrees of growth of finitely generated groups and the theory of invariant means, Izv. Akad. Nauk SSSR Ser. Mat. 48 (1984), no. 5, 939-985. MR 86h:20041

18. An example of a finitely presented amenable group that does not belong to the class $E G$, Mat. Sb. 189 (1998), no. 1, 79-100. MR 99b:20055

19. V. S. Guba and M. V. Sapir, On subgroups of the R. Thompson group $F$ and other diagram groups, Mat. Sb. 190 (1999), no. 8, 3-60. MR 2001m:20045

20. Victor Guba and Mark Sapir, Diagram groups, Mem. Amer. Math. Soc. 130 (1997), no. 620, viii+117. MR 98f:20013

21. P. Hall, Wreath powers and characteristically simple groups, Proc. Cambridge Philos. Soc. 58 (1962), 170-184. MR 25 \#3080 
22. W. Charles Holland, The characterization of generalized wreath products, J. Algebra 13 (1969), 152-172. MR 41 \#1884

23. W. Charles Holland and Stephen H. McCleary, Wreath products of ordered permutation groups, Pacific J. Math. 31 (1969), 703-716. MR 41 \#3350

24. Léo Kaloujnine and Marc Krasner, Produit complet des groupes de permutations et problème d'extension de groupes. I, Acta Sci. Math. Szeged 13 (1950), 208-230. MR 14,242b

25. Peter M. Neumann, On the structure of standard wreath products of groups, Math. Z. 84 (1964), 343-373. MR 32 \#5719

26. A. Ju. $\mathrm{Ol}^{\prime}$ šanskiı̌, On the question of the existence of an invariant mean on a group, Uspekhi Mat. Nauk 35 (1980), no. 4(214), 199-200. MR 82b:43002

27. Alexander Yu. Ol'shanskii and Mark V. Sapir, Non-amenable finitely presented torsion-bycyclic groups, Publ. Math. Inst. Hautes Études Sci. (2002), no. 96, 43-169 (2003). MR 2004f: 20061

28. Derek J. S. Robinson, A course in the theory of groups, 2nd ed., Graduate Texts in Math., vol. 80, Springer, New York, 1996.

29. Richard J. Thompson, 1973, Handwritten, widely circulated, unpublished notes attributed to Thompson, (c. 1973+).

30. Stan Wagon, The Banach-Tarski paradox, Encyclopedia of Mathematics and its Applications, vol. 24, Cambridge University Press, Cambridge, 1985, With a foreword by Jan Mycielski. MR 87e:04007

31. The Banach-Tarski paradox, Cambridge University Press, Cambridge, 1993, With a foreword by Jan Mycielski, Corrected reprint of the 1985 original. MR 94g:04005

Department of Mathematical Sciences

State University of New York at Binghamton

Binghamton, NY 13902-6000

USA

email: matt@math.binghamton.edu 